\title{
Diagnostic assays for leprosy based on T-cell epitopes
}

\author{
H. M. DOCKRELL,${ }^{1} *$ S. BRAHMBHATT,${ }^{1}$ \\ B. D. ROBERTSON, ${ }^{2}$ S. BRITTON ${ }^{3}$ U. FRUTH ${ }^{4}$ \\ N. GEBRE,${ }^{3}$ M. HUNEGNAW,${ }^{3}$ R. HUSSAIN,${ }^{5}$ \\ R. MANADHAR, ${ }^{6}$ L. MURRILLO, ${ }^{7}$ \\ M. C. V. PESSOLANI ${ }^{8}$ P. ROCHE, ${ }^{6}$ J. L. SALGADO ${ }^{8}$ \\ E. SAMPAIO ${ }^{8}$ F. SHAHID ${ }^{5}$ J. E. R. THOLE ${ }^{2} * *$ \\ \& D. B. YOUNG ${ }^{2}$
}

${ }^{1}$ Immunology Unit, Department of Infectious and Tropical Diseases, London School of Hygiene \& Tropical Medicine, London, UK, ${ }^{2}$ Infectious Diseases and Microbiology, Imperial College School of Medicine, St Mary's Campus, London, UK, ${ }^{3}$ Armauer Hansen Research Institute, Addis Ababa, Ethiopia, ${ }^{4}$ Vaccines and Biologicals, World Health Organization, Geneva, Switzerland, ${ }^{5}$ Microbiology Department, Aga Khan University, Karachi, Pakistan, ${ }^{6}$ Anandaban Leprosy Hospital, Kathmandu, Nepal, ${ }^{7}$ Instituto de Immunologia, Bogota, Columbia and ${ }^{8}$ Leprosy Laboratory, Oswaldo Cruz Institute, FIOCRUZ, Rio de Janeiro, Brazil

\begin{abstract}
Summary To date, only a limited number of antigens have been described as specific for Mycobacterium leprae, and in many cases, homologues have subsequently been shown to exist in mycobacteria such as $M$. avium and $M$. intracellulare. A Leprosy Synthetic Peptide Skin Test Initiative was established by the Steering Committee on the Immunology of Mycobacteria of the UNDP/World Bank/WHO Special Programme for Research and Training in Tropical Diseases, to investigate the potential of synthetic peptides that encode T-cell epitopes as diagnostic tools, which could be used to develop a skin-test reagent specific for leprosy. Such M. lepraespecific peptides should have unique amino acid sequences, or significant sequencedissimilarity from those in other mycobacteria. Synthetic peptides, 15 amino acids long, were synthesised from 33 genes or open reading frames within the M. leprae genome. Tuberculoid leprosy patients from four leprosy-endemic countries, Brazil, Ethiopia, Nepal and Pakistan, were tested as subjects known to have been infected with M. leprae, and to make good T-cell responses to antigens of $M$. leprae; UK blood donors were used as non-exposed or non-infected subjects. Peptides inducing potentially specific responses in leprosy patients and not in UK controls, and those
\end{abstract}

Correspondence to: H. M. Dockrell, Immunology Unit, Department of Infectious and Tropical Diseases, London School of Hygiene and Tropical Medicine, Keppel Street, London WC1E 7HT, UK

** Current address: Department of Bacteriology, ID-Lelystad, Lelystad, The Netherlands 
inducing cross-reaction responses, present in both leprosy patients and non-exposed, non-infected controls, were identified. A difference from the equivalent $M$. tuberculosis sequence of five or more amino acid residues did not, by itself, identify peptides that were $M$. leprae-specific, suggesting that many of these peptides may have homologues in environmental mycobacteria. To date, this approach has identified a number of peptides with greater than $90 \%$ specificity and $19-47 \%$ sensitivity, which are undergoing further specificity-testing. Such peptides would have great potential as T-cell reagents with which to monitor exposure to M. leprae within communities, formulated either as skin-test reagents, or as antigens for tests in vitro.

To be specific for Mycobacterium leprae, a peptide epitope must have a unique sequence of amino acids, not found in other mycobacteria or other infectious agents, or a significant degree of sequence-dissimilarity from the equivalent sequence in other mycobacteria. The peptide should be recognized by $\mathrm{T}$ cells from subjects known to be infected with $M$. leprae, such as patients with tuberculoid leprosy, and should not be recognized by those infected with other mycobacteria such as M. tuberculosis. M. leprae-specific peptides could be used to confirm early cases of leprosy, in whom diagnosis is often difficult, but would not be useful for the diagnosis of patients with multibacillary lepromatous disease, as these individuals mount weak or negligible T-cell responses. The peptide should also be recognised more strongly by $\mathrm{T}$ cells from subjects living in areas highly endemic for leprosy than by $\mathrm{T}$ cells from those living in areas of low prevalence. If such $M$. leprae-specific peptides were identified, they could be used to monitor the degree of exposure to leprosy within different communities, to aid leprosy control programmes to target their efforts in areas in which contact with $M$. leprae is more common.

Peptides 15 amino acids long were selected, using FINDPATTERNS and Epimer, ${ }^{1}$ from the genome of $M$. leprae, based on the presence of three or more HLA-DR binding-motifs, and synthesized. Peptides that bind to more than one HLA type would make better diagnostic reagents, as they would have the ability to be presented by antigen-presenting cells in a larger proportion of the population. ${ }^{2,3}$ However, even using such genetically permissive or 'promiscuous' peptides, it is likely to be necessary to use a pool of peptides, to ensure that all individuals from different ethnic groups, with different HLA types, could respond.

Tuberculoid leprosy patients were used as a group of subjects known to be infected with $M$. leprae, and known to make good T-cell responses to $M$. leprae and its antigens. Healthy staff members, who had worked closely with leprosy patients over many years without developing the disease, were also used, as such subjects, who are actually contacts to patients with leprosy, are known to show very strong T-cell responses. The leprosy patients and contacts were recruited in four leprosy-endemic countries, Brazil, Ethiopia, Nepal and Pakistan, in order that peptides capable of inducing T-cell responses in different ethnic groups might be identified. As non-exposed subjects, blood-bank donors in the UK were used, as it is extremely unlikely that any of these subjects would have visited a leprosy-endemic country, or have been exposed to M. leprae.

To date, 81 peptides from 33 genes have been tested for their ability to induce lymphocyte proliferation and secretion of interferon- $\gamma(\mathrm{IFN} \gamma)$ in standard human peripheral blood monunuclear cell (PBMC) cultures. ${ }^{4}$ Although most cultures showing good IFN $\gamma$-production also gave a proliferative response, secretion of $\mathrm{IFN} \gamma$ proved a more sensitive assay for detecting T-cell responses to peptides than did lymphocyte proliferation. This is likely the result of accumulation of IFN $\gamma$ over the 6-day assay period, whereas lymphocyte proliferation is measured only overnight, the cultures being pulsed with tritiated thymidine on day 5 . 
Intracellular cytokine staining was performed in peptide-stimulated cultures from UK subjects to confirm that the majority of the cells making IFN $\gamma$ were T cells.

Peptides showing greater responses in the M. leprae-infected or exposed groups than in the UK non-exposed group were identified, but other peptides gave equivalent responses in both groups. ${ }^{4}$ Analysis of the peptide sequences indicated that many of the peptides inducing good T-cell responses in the non-exposed UK donors had similar sequences in M. leprae and M. tuberculosis. Comparison of the 15 peptides tested to date which differed by five or more amino acid residues from those in the equivalent $M$. tuberculosis sequence showed that eight peptides had a specificity of $90 \%$ or greater, as defined by the IFN $\gamma$-test in the UK nonexposed group. ${ }^{4}$ The sensitivities to these peptides varied, usually being greater among leprosy-contacts than among tuberculoid leprosy patients. No peptide induced positive responses in all of the $M$. leprae-infected or exposed subjects, so that a number of peptides would need to be pooled to provide a reagent capable of inducing T-cell reactivity in the majority of subjects of any ethnic group. It was interesting that a difference of five or more amino acids residues from those in $M$. tuberculosis did not by itself predict $M$. leprae specificity; although the position of such amino acid changes relative to the T-cell contact residues will be critically important, it is also possible that there may be homologous epitopes within other mycobacteria such as $M$. avium, for which the full genome sequence is not yet known. Many of the peptides that induced the strongest T-cell responses in both M. lepraeinfected and exposed subjects as well as the UK controls had very similar or identical sequences in $M$. le prae and M. tuberculosis; this suggests that T cells may have been strongly primed or boosted to such epitopes by vaccination with $M$. bovis BCG, or by contact with the equivalent conserved epitopes within environmental mycobacteria.

One finding of interest was the detection of strong T-cell responses to peptides from open reading frames or genes of $M$. leprae not previously known to be T-cell antigens. This suggests that the repertoire of T-cell antigens recognized during $M$. leprae infection may be much broader than had previously been thought. Many of these genes are predicted to encode enzymes involved in housekeeping or general metabolic functions, genes the structure of which might be relatively conserved. Ongoing testing of the lead peptides identified to date will include their testing with PBMC from patients with pulmonary tuberculosis; ideally, testing with PBMC from patients infected with other mycobacteria such as $M$. avium should also be performed. The peptides must also be tested with PBMC from control subjects in the various leprosy-endemic countries.

The $M$. leprae genome is now thought to contain as many as 100 genes not present in $M$. tuberculosis, which might therefore be $M$. leprae-specific genes. ${ }^{5}$ Peptides from these sequences should therefore be tested as a high priority. The current approach, using the presence of multiple HLA-DR binding motifs, has proved to be capable of predicting peptides that induce T-cell responses in infected subjects. It may therefore be an efficient strategy to select for testing peptides containing HLA-DR binding motifs within M. leprae-specific genes. Another approach would be to make overlapping peptides that span the full sequences of these genes, or at least of selected genes. This approach may identify additional T-cell epitopes, which, however, might lack the ability to be recognized by $\mathrm{T}$ cells from subjects belonging to different ethnic groups. ${ }^{6}$ A third strategy would be to express the M. lepraespecific genes as recombinant antigens, and to map T-cell peptide epitopes only within those antigens that give the strongest and most specific responses.

In summary, the work performed to date by this strategic Task Force has shown that a multicentre, co-ordinated approach using peptides predicted to contain three or more HLA- 
DR binding-motifs can identify T-cell epitopes which are recognized by significant proportions of M. leprae-infected or exposed subjects. Further specificity testing of these peptides is now required, using samples of blood from patients infected with $M$. tuberculosis. A similar approach could be used to identify CD4 T-cell epitopes within genes present in M. leprae but absent from $M$. tuberculosis, which might have greater specificity. Such T-cell reagents could be used as research tools, to develop assays for early diagnosis, or to monitor exposure to $M$. leprae within leprosy-endemic countries.

\section{Acknowledgements}

This study was supported by a grant from the IMMYC Steering Committee of the World Health Organisation.

\section{References}

${ }^{1}$ Meister GE, Roberts CGP, Berzofsky JA, De Groot AS. Two novel T cell epitope prediction algorithms based on MHC-binding motifs; comparison of predicted and published epitopes from Mycobacterium tuberculosis and HIV protein sequences. Vaccine, 1995; 13: 581-591.

2 Harris DP, Vordermeier HM, Arya A et al. Permissive recognition of a mycobacterial T-cell epitope: localization of overlapping epitope core sequences recognised in association with multiple histocompatibility complex class II I-A molecules. Immunology, 1995; 84: 555-561.

${ }^{3}$ Mustafa AS, Lundin KE, Meleon RH et al. Identification of promiscuous epitopes from the mycobacterial 65 kilodalton heat shock protein recognised by human CD4(+) T cells of the Mycobacterium leprae memory repertoire. Infect Immun, 1999; 67: 5683-5689.

${ }^{4}$ Dockrell HM, Brahmbhatt S, Robertson BD et al. A post-genomic approach to the development of a M. leprae specific skin test. Infect Immun, 2000; in press.

5 Cole S. Preliminary analysis of the genome sequence of Mycobacterium leprae. Lepr Rev, 2000; 71: 162-164.

${ }^{6}$ Adams E, Britton W, Morgan A et al. Individuals from different populations identify multiple and diverse determinants on mycobacterial HSP70. Scand J Immun, 1994; 39: 588-596.

\section{DISCUSSION}

Professor Modlin: If I remember correctly, in our studies of the $10 \mathrm{kDa}$ antigen, we found that only two differences in the amino acid sequence of certain peptides determined specificity for M. leprae or M. tuberculosis. It might be that some peptides with fewer than five mismatches would be useful, if the mismatches occur in the area of the T-cell-receptor contact site.

Dr Dockrell: I agree. Only a single amino acid mismatch in a critical position might be enough to change the specificity of a peptide. The IMMYC-sponsored programme has an inventory of 110 peptides that have not yet been tested, and I have just spent some time in Stewart Cole's laboratory, seeking means of predicting activity and specificity.

Professor Britton: Do the peptides that you've tested include a range of HLA haplotypes? And have there been differences of reactivity to the peptides in different populations? We found a number of peptides to which Australians reacted but Nepalese did not. One of the strengths of your approach is that you are testing the peptides on two different continents among a variety of population groups; this is the correct approach.

Dr Dockrell: Some of the peptides elicited a strong response in Brazil and not in Pakistan, or in Pakistan but not in Ethiopia. This approach presupposes the use of a pool of peptides with varying HLA specificity. Unfortunately, we have been unable to HLA-type donors in the 
leprosy-endemic areas. We did type the donors in the UK; however, the results were not very useful. Many with the corresponding HLA type responded, but not all, and some with another HLA type responded.

Dr Klatser: Can you explain the fact that some of the peptides elicit stronger reactions among contacts than among leprosy patients?

Dr Dockrell: Most of the contacts were staff employed in leprosy treatment centres for many years who have not become ill. Such individuals have been noted by many workers to have very strong T-cell responses.

Dr Kaplan: You don't know which sub-set of lymphocytes responds. NK cells produce a great deal of IFN $\gamma$, which will remain in the supernatants for 6 days. The early NK-cell response from the normal donors would be detected in your system, and be indistinguishable from an antigen-specific response.

Dr Dockrell: In the UK, we have examined the T-cell profile with intracellular cytokine staining. The responding cells appear to be CD4. I believe that strong responses represent T-cell responses; I agree, however, that, in the case of weaker responses, we may be measuring a contribution by NK cells. Because not all donors respond, one must attribute the response to T-cells or invoke antigen-specificity on the part of NK cells.

Dr Kaplan: The cultures accumulate IFN $\gamma$ for the 6 days of the incubation, during which the IFN $\gamma$ does not decay. Intracellular cytokine staining shows the cellular profile at the moment. Depending upon the time at which you carry out the staining, you could measure IFN $\gamma$ produced by cells that are no longer present in the culture.

Professor Modlin: Dr Kaplan, do you suggest that the peptides stimulate the NK cells to produce IFN $\gamma$ ?

Dr Kaplan: NK-cells are stimulated to produce IFN $\gamma$ by cytokines IL-2, IL-12, IFN $\gamma$ and TNF that are produced by bystanding T-cells and monocytes. Analysis by FACS has shown that, at certain intervals, as many as $50 \%$ of the IFN $\gamma$-producing cells are CD3-minus.

Professor Modlin: In the final analysis, then, it is antigen-specific T-cells that trigger a non-specific NK-cell response.

Dr Kaplan: Production of IL-12 by monocytes is not antigen-specific. My point is that one must seek an explanation for the observation that individuals whom we suppose not to be sensitized respond strongly. I contend that we simply don't know which cell is responding.

Dr Dockrell: In the situation of UK blood donors who respond strongly to some of the peptides, we are not certain that the peptides are specific to M. leprae. 